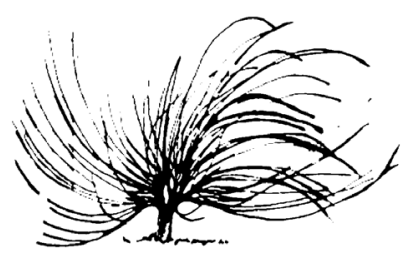

\title{
Modelo institucional de la orientación en el sistema educativo público costarricense
}

\author{
Alejandra Gamboa Jiménez ${ }^{1}$ \\ Universidad Nacional \\ Costa Rica \\ alejandra.gamboa.jimenez@una.cr \\ Manuel Arturo Fallas Vargas
Universidad Nacional
Costa Rica
manuel.fallas.vargas@una.cr \\ Shirley Ramírez Mora ${ }^{3}$ \\ Ministerio de Educación Pública \\ Costa Rica \\ shirley.ramirez.mora@mep.go.cr
}

\section{Resumen}

Este artículo contempla reflexiones con respecto al modelo disciplinar de la orientación en el sistema educativo

\section{(c) $(\Phi \otimes \Theta$}

Recibido: 26 de febrero de 2020. Aprobado: 2 de setiembre de 2020.

http://dx.doi.org/10.15359/rep.16-1.13

1 Orientadora, catedrática por la Universidad Nacional, Costa Rica y Doctora en Ciencias Sociales por la Universidad Nacional, Costa Rica. Magíster en Psicopedagogía y con estudios de grado en Ciencias de la Educación con énfasis en Orientación. Coordinadora del Proyecto de Investigación Modelos de Orientación, División de Educación para el Trabajo, Centro de Investigación y Docencia en Educación.

2 Orientador, catedrático e investigador del Proyecto de Investigación Modelos de Orientación, Doctor en Sexualidad y Relaciones Interpersonales por la Universidad de Salamanca (España), Magíster en Psicopedagogía, Licenciado en Ciencias de la Educación con énfasis en Orientación.

3 Orientadora, asesora nacional de Orientación, Departamento de Orientación Educativo y Vocacional del Ministerio de Educación Pública. Máster en administración Educativa, Universidad Latina. Licenciada en Derecho, Universidad Federada de Costa Rica. Licenciada y Bachiller en Orientación de la Universidad Nacional. 
público, un análisis dentro del marco del proyecto Modelos de orientación desde una mirada del perfil de desempeño profesional de la Universidad Nacional, Costa Rica. Un sistema, destacado por la diversidad de centros, modalidades, niveles y las múltiples características de la población que atiende. Metodológicamente, se utiliza el diseño fenomenológico, contempla tanto las entrevistas a personas claves del contexto en estudio y se hace uso de la triangulación para entrelazar los datos empíricos y los perfiles de contratación, para lo cual el empleo de la herramienta AtlasTi, contribuye en la organización de los datos. Se concluye, que el modelo institucional de orientación educativa en el Ministerio de Educación Pública (MEP), se intersecciona entre el andamiaje jurisprudencial que conforma el accionar de las modalidades, niveles y el contexto específico de cada centro educativo y las demandas del ejercicio profesional tanto por el cumplimiento de los lineamientos técnicos emanados del MEP, como por aquellas que surgen producto de las situaciones emergentes disciplinares de la cotidianidad laboral.

Palabras clave: Orientación educativa, orientación en escuelas, orientación en secundaria, modelos de orientación, funciones de orientación, desempeño profesional en orientación y sistema educativo.

\begin{abstract}
This article includes reflections on the disciplinary model of Educational Counseling in the public educational system of Costa Rica, an analysis within the framework of the project Counseling Models from the Viewpoint of the Professional Performance Profile of the Universidad Nacional, Costa Rica, a system highlighted by the diversity of centers, modalities, levels, and the multiple characteristics of the population it assists. Methodologically, the phenomenological design is used, contemplating both interviews with specific people in the context under study and triangulation to interlink empirical data and hiring profiles, for which the use of the tool AtlasTi tool contributed to the organization of the data. It is a conclusion that the institutional model of Educational Counseling in the Ministry of Public Education (MEP, for its acronym
\end{abstract}


in Spanish) is intersected between the jurisprudential scaffolding that shapes the action of the modalities, levels, and specific context of each educational center and the demands of professional practice both for compliance with the technical guidelines issued by MEP and those that arise from emerging disciplinary situations of everyday work.

Keywords: educational counseling, school counseling, high school counseling, counseling models, counseling functions, guidance functions, professional performance in counseling and educational system

\section{Introducción}

$\mathrm{E}$ ste estudio plantea diversas reflexiones que conllevan al análisis de la orientación dentro del sistema educativo público costarricense, con base en el desempeño profesional, las funciones dictadas por el Servicio Civil y los lineamientos técnicos emanados del MEP (Ministerio de Educación Pública, 2019). El estudio aquí presentado forma parte de uno mayor, correspondiente a una investigación realizada en la Universidad Nacional, Costa Rica, sobre modelos institucionales en la disciplina de la orientación. Con ello, se configura un referente sobre los modelos de orientación aplicados en las instituciones costarricenses que, históricamente, han nutrido la identidad profesional por medio de un accionar consolidado desde la jurisprudencia, con su apoyo por más de cincuenta años. A su vez, estos han ido evolucionando según los cambios en los modelos económicos y productivos mundiales, e impregnan la actual práctica profesional en orientación.

\section{Marco referencial}

La comprensión del modelo institucional constituido de la orientación en el sistema educativo público se enmarca desde la mirada histórica de este escenario a nivel nacional, además se contemplan las funciones adscritas al desempeño profesional mediante las dictadas por el Manual de puestos del Servicio Civil (Dirección General de Servicio Civil, 2020) que derivan en el título I y II de los cuales emanan funciones específicas para cada puesto y otros aspectos de la jurisprudencia institucional. Por supesto, estas inciden en el abordaje orientador. 


\section{Antecedentes históricos y el camino hacia la profesionalización}

En Costa Rica, el desempeño disciplinar de la orientación data de 56 años de experiencia, desde su incorporación en escenarios laborales, los cuales son respaldados por la legislación nacional e internacional, por ejemplo, el Convenio Centroamericano de Educación (Asamblea Legislativa, 1966) en su Artículo 7, el cual, a su vez, le ha permitido su constante profesionalización.

La legitimación del espacio laboral de orientación en el sistema educativo le ha permitido el largo recorrido histórico remontado al año 1957, mediante la incorporación de los servicios de orientación en la Ley Fundamental de Educación (Asamblea Legislativa, 1957). Desde esta data se ha fortalecido el accionar del colectivo de profesionales en orientación; en un principio con fines prospectivos de apoyo al estudiantado. Posteriormente, el Convenio Centroamericano sobre la Unificación Básica de la Educación N.³726 (Asamblea Legislativa, 1966), legitima la orientación escolar y vocacional, y aunque en sus inicios los recursos teóricos eran limitados, a través de los aportes de otras disciplinas, principalmente la pedagogía, la psicología y la sociología, se ha consolidado un conglomerado teórico y metodológico, lo cual permite establecer las bases epistemológicas de la disciplina en el país.

El camino de la profesionalización no ha cesado desde sus inicios $\mathrm{y}$, dichosamente, existe una conformación disciplinar en este contexto. La historia también permite concluir los diferentes altibajos de la orientación en el MEP, en términos de robustecer el número contratado de profesionales, es decir, el enfrentamiento a los retos del nivel presupuestario, los de visión disciplinar a largo plazo y los liderazgos políticos de turno, comprometidos en cada década, mayor o menormente, hacia la labor orientadora en diferentes periodos del pasado.

En sus inicios, la idea general de la orientación educativa fundamenta las bases de su especialización en las principales universidades públicas costarricenses, impulsada inicialmente por la Universidad de Costa Rica (UCR) con la carrera de Ciencias de la Educación con énfasis en Orientación y luego la Universidad Nacional. Con ello, el colectivo profesional especializado en la disciplina, desde el nivel de grado, logra ubicarse en el mercado laboral, principalmente por el Ministerio de Educación Pública, el cual nombra a la mayoría de profesionales graduados y graduadas de esta carrera. Asimismo, en el 
camino de profesionalización, la UCR ha consolidado un Posgrado en Educación con énfasis en Orientación, con lo cual se contribuye, desde el nivel superior, con idoneidad disciplinar y profesional. Más recientemente, con el apogeo de la educación superior privada, otras casas de enseñanza vieron su auge económico en la formación de profesionales en esta área.

Pasado este medio siglo, se ha experimentado un cambio generacional, producto de las jubilaciones de las personas pioneras en la profesionalización de la disciplina. Además, el Estado costarricense, como ocurre en casi todas las naciones, ha venido transformándose, producto de las influencias externas y demandas del contexto global, las cuales marcan un itinerario y desafíos que han alcanzado, de manera directa, el sistema educativo.

\section{Modelo institucional de la orientación en el MEP}

Existen diferentes modelos de orientación; de la bibliografía se pueden extraer aquellos relacionados con la intervención (Monescillo, Méndez y Bisquerra, 2008 y 2011), los vocacionales, ocupacionales, profesionales y laborales (Müller, 1998 y 2004), los psicologistas (Naranjo, 2004a y 2004b). Sin embargo, en este estudio es imperante la comprensión de los modelos institucionales en los tres escenarios, en los cuales, a través de la historia, se han contratado constantemente profesionales de la orientación, es decir, campos laborales consolidados, tales como el sistema penitenciario, la educación técnica, a través del Instituto Nacional de Aprendizaje y el más grande empleador que es el MEP, en el que se fundamenta este artículo.

Ahora bien, ¿qué significa un modelo institucional en orientación? Para Gamboa y Fallas (2019, p. 10) lo siguiente:

El modelo institucional se concibe como aquella representación institucional, que basa su accionar en un sistema de interacciones donde confluyen dinámicas endógenas y exógenas que permean al sistema donde se desarrollan los servicios de Orientación, que en muchos de los casos se desenvuelven mediante el trabajo interdisciplinario con acciones de carácter educativo, preventivo, de investigación y de desarrollo humano. El modelo está vinculado mediante acciones planificadas y emergentes; a partir de la jurisprudencia que dicta el orden estatal. 
En el Ministerio de Educación Pública, se encuentra un texto complejo donde se resalta el Modelo de intervención de orientación en el sistema educativo costarricense (Carvajal, Estrada, Mora y Vargas, 2014), donde se referencian aspectos del modelo institucional de la disciplina en forma de texto orientador y no de aplicación obligatoria; rescata puntos de vista globales y caleidoscópicos de la intervención orientadora en educación y permite que se cumplan los itinerarios profesionales de la disciplina y las demandas de cada institución. Sin embargo, las políticas, funciones y lineamientos que requiere conocer y aplicar cada profesional sí son obligatorios:

Cada institución tiene sus propias dinámicas laborales, estatutos $\mathrm{y}$ formas de cumplir con sus objetivos, a diferencia de las organizaciones, las instituciones estatales se configuran entre una red de jurisprudencia respaldada en su accionar desde las políticas del Estado, esto constituye la plataforma del modelo institucional. De esta premisa se diferencia la categoría modelo institucional del modelo organizacional, ésta última responde a fines internos con regulaciones independientes del Estado. (Gamboa y Fallas, 2019, p. 10)

Los modelos institucionales corresponden a una categorización poco explorada en la comunidad científica, en comparación con los de intervención en el área de la orientación; sin embargo, en el contexto español se encuentra un hallazgo acotado a los intereses del presente estudio y son Grañeras y Porras (2008), quienes señalan que los modelos institucionales son estructuras organizativas provistas de recursos establecidos por las administraciones para implementar los principios de la orientación y, es en la práctica donde esas estructuras se consolidan y legitiman, otorgándole pertenencia institucional por sus funciones diferenciales. Esta definición del contexto educativo español permite un marco de referencia contextualizado en los sistemas más tradicionales donde se hace orientación.

El MEP señala un modelo organizativo institucional del servicio de orientación, que enmarca la estructura nacional, regional e institucional, la cual, por su secuencia organizativa y jerárquica, permite comprender su integralidad. Se describe de la siguiente forma: 
Bajo la figura de un Departamento Central de Orientación es la encargada de emitir las directrices y lineamientos relacionados con el quehacer profesional de las poblaciones que conforman el sistema, a saber: Asesorías Regionales, Departamentos de Orientación de III ciclo y Educación Diversificada, Equipos Interdisciplinarios de Escuelas de Atención Prioritaria, Profesionales de Orientación de las Escuelas de Excelencia y Horario Ampliado, y Comités de Orientación en primaria. (Carvajal, Estrada, Mora y Vargas, 2014, p. 22)

El nivel macro del Departamento de Orientación Educativa y Vocacional (DOEV) del MEP se encuentra adscrito a la Dirección de Vida Estudiantil, a esta dirección pertenecen, además, los Departamentos de Salud y Ambiente, el Departamento de Convivir y el Departamento de Participación Estudiantil, los cuales comparten labores entre ellos. Asimismo, el DOEV coordina acciones con las instancias pertenecientes a la Dirección de Desarrollo Curricular.

A partir de lo anterior, se deduce cómo la organización jerárquica del escenario en estudio permite comprender, a través de un ente central, la organización de las acciones ejecutables en los últimos eslabones, es decir, los centros educativos, concretamente en el servicio profesional especializado de orientación. Asimismo, en este nivel, se conjugan las coordinaciones entre las personas profesionales de los centros y los actores que en él convergen, entre ellos: la específica con el estudiantado y su familia, el personal docente, la gestión administrativa de la institución, consejos institucionales, comités ligados al accionar orientador: convivir, apoyo educativo, transporte estudiantil, bienestar estudiantil --becas--, servicio comunal estudiantil, Unidad de Permanencia y Reincorporación Educativa 1 (UPRE) u otras. Además, los proyectos o programas adscritos al centro educativo marcan un accionar específico y las acciones de la persona profesional en orientación, ejemplos de ellos son: Prevención de Violencia, UPRE-PROEDUCA, Iniciativa Salud Mesoamérica (ISM-Salud Mesoamérica), Talleres de verano, Ciencia Tecnología, Arte y Matemática, conocido por sus siglas en inglés como STEAM, Colegios de Alta Oportunidad, entre otros. En conclusión, se encuentran dos niveles de comprensión del modelo institucional de la orientación en el MEP, uno macro que corresponde a las políticas y regulaciones estatales y ministeriales; además un 
nivel micro, donde corresponden las acciones cotidianas del desempeño profesional, que incluyen una serie de programas y proyectos que involucran su accionar.

Esta labor representa una tarea compleja en cuanto a la coordinación y rendición del trabajo en diferentes niveles de jerarquías, que demandan sincronicidad de acciones, complementariedad de trabajo y conexión interdisciplinaria. Las múltiples interacciones que surgen entre el trabajo orientador producen una serie de procesos y vinculaciones que dan sentido al modelo institucional en orientación.

Para ello, dicho modelo se fundamenta en la premisa sistémica, donde convergen tres subsistemas de orientación: el institucional, el regional y el nacional. A su vez, el institucional posee otros niveles de intervención más, a saber, la familia, la comunidad y otros agentes. Estos tres, igualmente, poseen niveles de rendición y retroalimentación del abordaje orientador, que convergen con las áreas de intervención, calificadas como orientación individual, colectiva y asesoramiento; todas ellas abordan el conocimiento de cada persona en sí misma, del medio, la toma de decisiones y el compromiso social (Carvajal, Estrada, Mora y Vargas, 2014).

En el modelo como representación de una realidad específi$\mathrm{ca}$, intervienen elementos propios de su contexto, lo cual permite la comprensión del escenario específico, ya que los modelos son "representaciones, en este caso de la realidad, explicando y justificando qué se entiende por realidad-objeto de intervención. En suma, el modelo resulta ser un instrumento útil que explica y justifica una serie acciones para intervenir en la práctica" (Arencibia, 2012, p. 82). Por ello, el modelo en el contexto en estudio reconoce, en sus subsistemas, cuatro pilares significativos: ubicar a sus profesionales en una vía específica, es decir las acciones profesionales deben corresponder a que las personas orientadas se conozcan a sí mismas, conozcan el medio en el cual se desarrollan, tomen decisiones y lo hagan con compromiso social-ecológico, como procesos del desarrollo educativo y vocacional.

El Ministerio de Educación Pública (2017a) determina el objeto de estudio de la orientación en el sistema educativo costarricense en los siguientes términos:

La potenciación del desarrollo integral de la población estudiantil, mediante procesos que contribuyen al autoconocimiento, 
conocimiento del medio, toma de decisiones con compromiso personal-social, para la planificación de la vida (sentido, estilo y proyectos), con el propósito de vincular el desarrollo personal (unicidad del ser) y de colectivos sociales (particulares), con el desarrollo social y económico del país y la sociedad planetaria, para la autorrealización de la persona y el bienestar común.

Se fundamenta esencialmente en la intervención continua, mediante los principios de prevención, desarrollo e intervención social desde el marco del proceso educativo, esto demanda asesorar al personal del centro educativo respecto a la educación integral como parte de su rol formador, así como a padres, madres o personas encargadas en su labor protectora y formadora.

El cuerpo de conocimientos teóricos, epistemológicos, principios científicos, procesos metodológicos e instrumentales, que fundamentan la planificación, el diseño, la aplicación y la evaluación de las intervenciones de las personas profesionales en Orientación, las cuales tienen como finalidad potenciar el desarrollo integral de la población estudiantil. (p. 1)

La amplitud del objeto de estudio requiere de vinculaciones que suplan las necesidades básicas que potencien a las personas, tomando como vértice la centralidad en el sujeto orientando, trascendiendo del entorno educativo a niveles comunitarios y sociales. Su labor contempla la interdisciplinariedad y depende de las necesidades del contexto que envuelve la institución; todo ello en coordinación entre docentes, gestores de la educación, instituciones colaboradores tales como, Equipos Básicos de Atención Integral en Salud (EBAIS), Caja Costarricense de Seguro Social (CCSS), Patronato Nacional de la Infancia (PANI), Poder Judicial, Instituto sobre Alcoholismo y Farmacodependencia de Costa Rica (IAFA), universidades y otros centros de formación para jóvenes y personas adultas, así como organizaciones de diferente naturaleza: las empresas, fundaciones, organizaciones comunales, entre otras.

Es decir, el modelo institucional en orientación tiene su accionar a partir del desarrollo integral de sus estudiantes, lo cual implica un gran desafío en el desarrollo profesional en la cotidianidad, con parámetros de acción definidos mediante tres componentes de intervención 
definidos por el MEP, a saber: el vocacional, el personal social y el educativo; todos desde la prevención como primer propósito. Asimismo, dicho modelo debe responder a los Programas de Estudio de Orientación (2017), los cuales incluyen el Primero, Segundo y Tercer ciclos de la Educación General Básica y Educación Diversificada.

[Estos programas] establecen procesos de orientación colectiva desde primaria hasta secundaria, dirigido al desarrollo de habilidades y valores en la población estudiantil, según su etapa de desarrollo, de manera, que, al finalizar dichos procesos, logren alcanzar un nivel competente y proficiente en su desarrollo cognitivo-educativo, vocacional y personal-social. (Ministerio de Educación Pública, 2017a, p. 7)

Toma en cuenta las ofertas y servicios del Sistema Educativo Costarricense, cuya estructura es variada de acuerdo con las múltiples necesidades: Educación Preescolar, Educación I y II Ciclos, Educación de III ciclo de la Enseñanza General Básica y Educación Diversificada Académica, Educación Técnica, Educación de personas jóvenes y adultas, Servicios de Educación Especial (Ministerio de Educación Pública, 2017c).

A partir de lo señalado, y por la complejidad que representa la labor orientadora en el sistema educativo, a continuación se abordan los subsistemas construidos para la comprensión del modelo institucional de la orientación en el MEP; estratégicamente, se desarrollan a partir del subsistema denominado Profesionales de la Orientación en Centro Educativo, el cual es el más relevante, no solo porque representa mayor cantidad de profesionales de la orientación en sus funciones, sino porque en él se cristaliza el fin último de la labor orientadora, es decir, el estudiantado. En este se explican las dinámicas medulares del accionar orientador para proseguir con el subsistema regional y terminar con el nivel nacional, tomando en consideración la propia concepción establecida por el DOEV en su organización, la cual está constituida en el ámbito central por el DOEV, en el ámbito regional por las Asesorías Regionales de Orientación (ARO), en los centros educativos por las personas profesionales del Servicio de Orientación, Comités de Orientación (primaria), los Departamentos de Orientación de III ciclo y en Educación Diversificada, docentes guías en secundaria (Ministerio de Educación Pública, 2019). 


\section{Subsistema: Profesionales de la orientación en el centro educativo}

La comprensión de la organización y funcionamiento del subsistema institucional está supeditado a la oferta educativa, la cual en Costa Rica es ampliamente diversa. Al respecto, cabe rescatar que no en todos los centros escolares se ofrecen los servicios de orientación, debido a cuestiones presupuestarias estatales, aun cuando la Ley Fundamental de Educación ampara la cobertura de este servicio. Como es generalizado a nivel global, Costa Rica posee educación preescolar, primaria y secundaria. Sin embargo, no es posible comprender el subsistema sin conocer la especificidad caracterizada a cada institución que desarrolla el servicio profesional especializado de orientación y que, en términos descriptivos, se pueden encontrar en el Compendio de la ofertas y servicios del Sistema Educativo Costarricense (Ministerio de Educación Pública, 2017c).

En la actualidad, el MEP contrata 1394 profesionales de la orientación entre profesionales del título I y II, cada cual con sus funciones específicas. Según los datos suministrados por este Ministerio para el 2020, por parte de la Unidad de Seguimiento de la Dirección de Recursos Humanos del MEP y, más específicamente, los datos que maneja el DOEV refieren que la mayoría de puestos en el área de orientación se ubican en los centros educativos de secundaria (1084), en segundo lugar la secundaria técnica y en tercer lugar en escuelas primarias de horario ampliado.

En el contexto del centro educativo, cada profesional de la orientación se ajusta a las demandas de la realidad institucional, esta a su vez es diversa, de acuerdo con sus características, por ejemplo: las escuelas de horario ampliado, por lo general, tienen solo una persona profesional de la orientación, sin apoyo de equipo interdisciplinario (psicología y trabajo social o sociología, según corresponda) que distinguen a otros tipos de escuelas. Otras diferencias corresponden a las particularidades en la oferta académica, ya sea esta técnica, nocturna, bilingüe, subvencionada, entre otras. Asimismo, los colegios académicos pueden presentar especialidades permeando la unicidad en la acción orientadora, tal es el caso de los Centros Integrados de Educación para Adultos (CINDEA), Institutos de Educación Comunitaria (IPEC), Colegios indígenas, Liceos rurales, Colegio Innovación Tecnológica, Ecológico, Deportivo. Es decir, la oferta educativa ofrecida en cada centro educativo marca la 
misión que debe contemplar la perspectiva orientadora para direccionar su quehacer.

En este subsistema de ordenamiento institucional, la diversidad de estrategias del servicio de orientación, tanto en primaria como en secundaria, debe contemplar tres condiciones: la primera es si en la institución labora sola una persona profesional de la orientación -condición recurrente principalmente en escuelas-; la segunda donde igual que la primera, solo labora un o una orientadora, con la salvedad de trabajar en conjunto con un equipo interdisciplinario (psicología, trabajo social o sociología, según corresponda) -condición exclusiva de la escuela primaria-, debido al abordaje en zonas con alta vulnerabilidad social; la tercera, cuya condición es la labor con más de una persona profesional de la orientación, donde uno o una posee la función de coordinación de los denominados Departamentos de Orientación, condición exclusiva de secundaria.

En concordancia con lo anterior, las estrategias de la primera condición, establecidas por la priorización de necesidades que presente la población que se atiende reflejan el accionar, pues existe una tendencia a trabajar con grupos de iguales que presentan características específicas, situaciones individuales y familiares. En la segunda condición, se espera un trabajo interdisciplinario de abordaje de las distintas situaciones escolares, ya sea atendiendo una situación particular con uno o una especialista del equipo, o bien, cuando la situación amerite la intervención conjunta. La tercera condición, por la naturaleza de las funciones y la obligatoriedad de impartir clases dentro del currículum escolar, existe la orientación colectiva, la atención individual, ya sea porque el estudiantado requiere del servicio de orientación por su propia voluntad, por remisión del colectivo docente, por solicitud de sus personas encargadas, madres o padres de familia, o cualquier otra situación; asimismo, la labor orientadora en algunos escenarios también hace uso de la orientación grupal, donde situaciones comunes son intervenidas en pequeños grupos de iguales.

Específicamente, en la educación secundaria se asigna un número de estudiantes que debe atender la persona profesional de la orientación, esto es conocido en el DOEV como ratio en orientación (Ministerio de Educación Pública, 2011), dicha condición aplica para aquellas instituciones donde hay Departamentos de Orientación, es decir, donde solo existe la contratación de un o una profesional. 
En la Tabla 1, se expone el referente para designar el número requerido de profesionales de la orientación, según las categorías de orientador y orientador asistente. Se evidencia la correlación entre el número de estudiantes y el número de profesionales de la orientación asignado; es decir, a mayor número de estudiantes, mayor número de profesionales se contratatan en el área; sin embargo, pese a ser mandatorio, es un punto de partida para la designación de profesionales, que si bien oficial, no en todos los centros educativos se puede cumplir, sobre todo por falta de recursos financieros estatales. Sin embargo, hay que tener presente que las implicaciones de los números que se reflejan en esta tabla se constituyen en retos cotidianos de la labor profesional, por la naturaleza de las funciones de la orientación.

\section{Tabla 1}

Recursos y asignación de profesionales de la orientación de acuerdo con el número de estudiantado que atiende

\begin{tabular}{|c|c|c|}
\hline \multirow{4}{*}{ 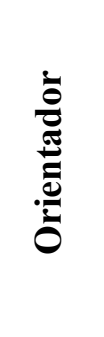 } & Rango de matrícula & Tabla de recursos \\
\hline & Hasta 500 estudiantes & $\begin{array}{l}1 \text { Categoría Orientador } 1 \\
\text { (Cód. 029-DHSC) }\end{array}$ \\
\hline & De 501 a 1000 estudiantes & $\begin{array}{l}1 \text { Categoría Orientador } 2 \\
\text { (Cód. 030-DHSC) }\end{array}$ \\
\hline & De 1001 estudiantes y + & $\begin{array}{l}1 \text { Categoría Orientador } 3 \\
\text { (Cód. 031-DHSC) }\end{array}$ \\
\hline \multirow{5}{*}{ 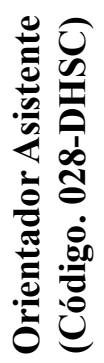 } & De 401 a 800 estudiantes & 1 Categoría Orientador asistente \\
\hline & De 801 a 1200 estudiantes & 2 Categoría Orientador asistente \\
\hline & De 1201 a 1600 estudiantes & 3 Categoría Orientador asistente \\
\hline & De 1601 a 2000 estudiantes & 4 Categoría Orientador asistente \\
\hline & De 2001 estudiantes y + & 5 Categoría Orientador asistente \\
\hline
\end{tabular}

Nota: Oficio DPI-DDSE-0296-11, del 03 de agosto de 2011, se emite por parte de la Dirección de Planificación Institucional del MEP. La categoría corresponde a las dictadas por el Dirección General de Servicio Civil (DGSC) de Costa Rica, ente regulador del empleo público Ministerio de Educación Pública, 2011.

En adición al párrafo supracitado, las funciones de la orientación tienen diferentes vertientes de implicación, las están en el orden 
jurídico, pero también en el orden integral de desarrollo de las personas a su cargo, cuyo volumen de trabajo es superado debido a las demandas propias del quehacer en orientación; solo para ofrecer un ejemplo en la categoría de personal técnico docente, donde se encuentran las personas profesionales de orientación que laboran en la educación secundaria, una de cuyas funciones es ejercer una acción directa y sistemática en la formación de la personalidad del estudiantado, que lo capacite para vivir conforme a los valores superiores del ser humano y la sociedad (Poder Ejecutivo, 1996). Asimismo, de esta función genérica emanan múltiples directrices para su operacionalización, tales como las implicaciones jurídicas del accionar (Fung-Lung, 2017) y las implicaciones relacionadas con el desgaste profesional de la labor en orientación (Gamboa, Álvarez, Flores, Miranda, y Vindas, 2018).

Todo ello es una condición exclusiva de profesionales que laboran en la educación secundaria que se catalogan, por el Estatuto de Servicio Civil, estipulado el 30 de mayo de 1953, mediante el título II de Carrera Docente. Este considera, dentro de su contratación, la variable técnico docente, no desde la típica mediación pedagógica de una materia del currículo, sino desde una visión holística tal y como se contempla en los programas de estudio dedicados a la orientación; es decir, en sus jornadas laborales deben dedicarse a ofrecer lecciones de orientación a los grupos a cargo, de forma semanal. Por el contrario, las personas profesionales en orientación que laboran en la educación primaria pertenecen al Título I del Estatuto en mención, es decir, al Régimen de Carrera Administrativa, como personal técnico-administrativo, cuyas implicaciones generales no requieren la obligatoriedad de la docencia en sus funciones.

A partir de lo anterior, se destaca que no existe el ratio en orientación en la educación primaria, específicamente, en las modalidades de centros educativos en cuya constitución se encuentran equipos interdisciplinarios, ni en las escuelas de horario ampliado y tampoco en las escuelas de excelencia. El modelo de contratación de la especialidad en orientación en educación primaria ha venido cambiando con el tiempo; en la actualidad, la dirección de los centros educativos puede hacer su solicitud al MEP para procurar la apertura del código de orientación, con la salvedad de tener el grado mínimo de licenciatura, dado que son contratos a partir del Título I que fue mencionado con anterioridad y la contratación surge de acuerdo con la especialidad profesional. Es 
por ello que la especialidad en orientación, atiende las necesidades de toda la población estudiantil y no tienen asignado lecciones o grupos, aunque no es una prohibición; de hecho, existen dinámicas de centros educativos donde se hacen trabajos colectivos de orientación también.

Se puede deducir que el número de estudiantes por cada profesional de la orientación varía, ante lo cual la atención a toda la población a cargo es improbable en términos de cobertura, aunque no por ello la labor deja de ser relevante y pertinente; lo que deja en evidencia que las estrategias del trabajo con la totalidad del estudiantado en cada centro educativo es único y representa un desafío en sí mismo, dado que el accionar orientador tiene dimensiones disciplinares, no solo de atención colectiva, sino también de atención individualizada de situaciones emergentes y de seguimiento, se une el trabajo interdisciplinario, se suman también la atención indirecta a miembros familiares lo que implica otro nivel de atención y el de orden social con organizaciones, ministerios e instancias exógenas, producto del trabajo con menores de edad (condición de la mayoría de centros educativos del MEP, pero no exclusiva).

Como se ha señalado anteriormente, el bagaje de acciones que representa el trabajo profesional en orientación es complejo y para ello una de las herramientas principales que existe para delimitarlo se denomina: Plan de Trabajo Anual (PAT), el cual es elaborado por cada departamento del centro educativo, este se diseña a partir de diferentes dispositivos, tales como los lineamientos del DOEV, que cada año se disponen mediante lo que se denomina "Orientaciones técnico-administrativas para operacionalizar el Servicio de Orientación en el ámbito regional y de centro educativo" (Ministerio de Educación, 2019), donde se incluye el accionar específico de la persona profesional en orientación y según sea su ámbito. Específicamente para secundaria, posee lineamientos para III ciclo y educación diversificada, tanto para los Departamentos de Orientación; para la jefatura técnica; para cada profesional de orientación y para docentes guía. Para primaria: existen lineamientos para I y II ciclos, tanto para profesionales del Servicio de Orientación y para los Comités de Orientación. Es decir, las funciones del colectivo profesional en orientación se fundamentan en el Manual Descriptivo de Puestos del Servicio Civil y se operacionalizan mediante las emitidas por el DOEV.

Dichos lineamientos abarcan el accionar profesional en orientación e incluyen, de forma general, los principios de la Política 
Educativa, que contempla "la persona: centro del proceso educativo y sujeto transformador de la sociedad" (Ministerio de Educación, 2019, p. 1) y el DOEV, por su parte, advierte sobre su sustento en el círculo de bienestar donde se concibe a la persona de forma multidimensional, con capacidad transformadora, cuya importancia se enmarca en el desarrollo vocacional. Además, como se ha mencionado, se lleva a cabo el trabajo con familias, debido a que la población meta, en su mayoría, son menores de edad; solo en aquellas modalidades donde la población escolarizada son las personas adultas, el énfasis del trabajo es distinto.

Finalmente, el DOEV coordina el Programa Guía y los Comités de Orientación, el primero tendiente al favorecimiento de funciones del docente guía "para el logro de procesos que contribuyen a potenciar habilidades, el desarrollo integral de la población estudiantil, la permanencia y el éxito escolar de su grupo a cargo; así como planteamiento de estilos y proyectos de vida" (Ministerio de Educación, 2019, p. 2), el segundo, tendiente al desarrollo integral del estudiantado, tiene su accionar en el I y II ciclos y en escuelas unidocentes con apoyo de la Asesoría Regional de Orientación y de la persona profesional de la orientación, si la hubiera. Es decir, con el fin de coadyuvar en el desarrollo integral del estudiantado, con este tipo de estrategias el servicio de orientación permea al sistema educativo: ante la carencia de códigos para el nombramiento de profesionales en esta disciplina, dichos programas pueden ser ejecutados por personal docente.

\section{Subsistema regional: La acción de las Asesoría Regionales de Orientación}

El contexto de las Asesorías Regionales Educativas del MEP en el país, corresponde a 27 Direcciones Regionales, distribuidas en las 7 provincias de Costa Rica (ver Figura 1), para cada una de ellas labora una persona profesional como asesora regional en orientación, es decir, un total de 27 asesores y asesoras regionales para todo el país. 


\section{Figura 1. Asesorías Regionales Educativas del Ministerio de Educación Pública, Costa Rica}

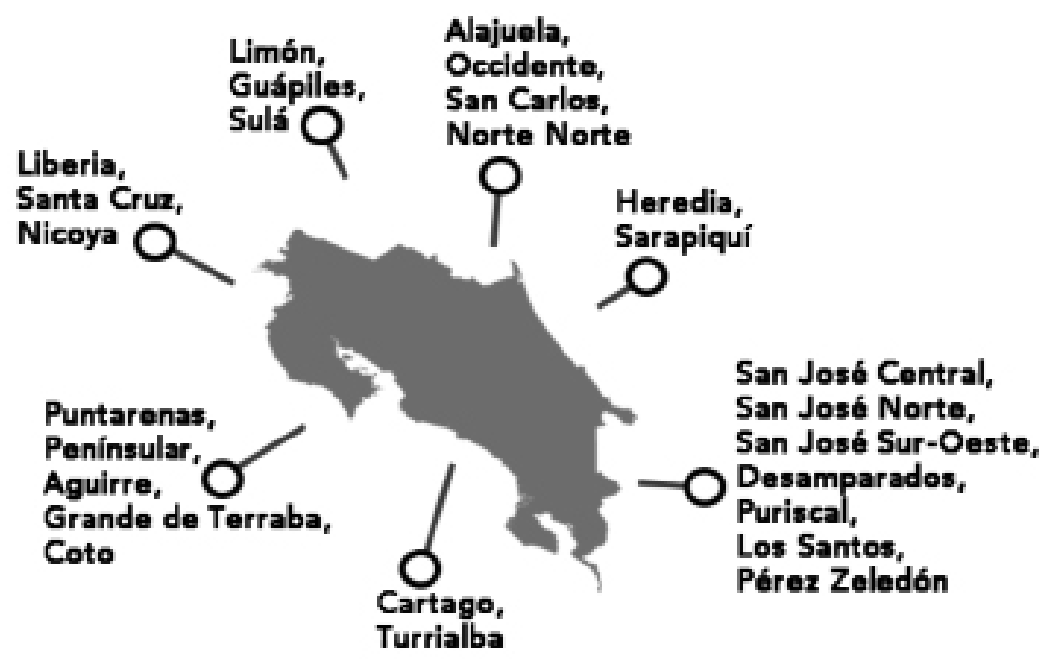

Nota: Elaborado a partir de los datos suministrados por el Departamento de Orientación Educativo y Vocacional. Lo indicado con asterisco $\left(^{*}\right)$, corresponde a aquellas asesorías regionales donde su profesional de la orientación es parte de un equipo interdisciplinar conformado además por profesionales de la psicología y trabajo social.

Las Asesorías Regionales de Orientación (ARO), al igual que el subsistema del centro educativo, basan su accionar en las Orientaciones técnico-administrativas para operacionalizar el servicio de orientación en el ámbito regional y de centro educativo (Ministerio de Educación, 2019). En este nivel, las personas profesionales de orientación atienden las necesidades de la región, además, desarrollan diferentes proyectos nacionales, tales como la estrategia contra la violencia, también las de índole vocacional, de permanencia del estudiantado en el sistema educativo, y todas aquellas que promueve el MEP; por medio de ellas se canalizan informes que tienen que presentarse a nivel nacional, para dar seguimiento al trabajo ejecutado por las personas profesionales de la orientación en el centro educativo.

Las ARO planifican asesoramientos regionales con su población meta en región, en algunas asesorías son mensuales y en otras tienen diferente periodicidad, con el fin de coordinar, brindar acompañamiento técnico y promover el trabajo orientador de acuerdo con las directrices y metas ministeriales. 


\section{Subsistema nacional: La acción de las asesorías nacionales}

Según el Ministerio de Educación (2019, p. 2), "el servicio de Orientación cuenta con una estructura organizativa desde el nivel central hasta centro educativo", de este nivel, se emanan las acciones y el cumplimiento de los aspectos legales y teórico-metodológicos que sustentan el servicio de orientación en el DOEV. La Asesoría Nacional de Orientación cuenta con seis personas profesionales en Orientación para cumplir con las funciones de la Asesoría Nacional de Orientación, las cuales prioritariamente son de organización técnico-administrativa para operacionalizar el servicio de orientación en el ámbito regional y de centro educativo y, por ende, es el ente que da cuenta a nivel ministerial del cumplimiento de metas y coordinaciones intraministeriales propias de su quehacer.

Este subsistema de orden jerárquico se rige a partir del soporte normativo y la jurisprudencia en general, que son dispositivos obligatorios que incluyen responsabilidades de la acción orientadora y permiten también comprender las dinámicas complejas de la orientación educativa, entre ellas los Programas de Estudio de Orientación primero, segundo y tercer ciclos de la Educación General Básica y Educación Diversificada (Ministerio de Educación Pública, 2017b) y el Decreto N. ${ }^{\circ}$ 38170-MEP, sobre la organización administrativa de las oficinas centrales del MEP, de donde se deriva jurisprudencia relacionada a la Dirección de Vida Estudiantil, ente superior a las Asesoría Nacionales del Departamento de Orientación Educativo y Vocacional (DOEV).

\section{Metodología}

Este estudio responde a un enfoque cualitativo. Como técnica de recolección de datos, se llevaron a cabo entrevistas semiestructuradas a tres personas profesionales de la orientación que laboran en el sistema educativo. Se combina el análisis de contenido de la jurisprudencia que contiene la labor de orientación en el contexto educativo y así conformar el estudio de caso, como diseño de investigación. Con esta estrategia metodológica, se recurre a la triangulación entre la indagación bibliográfica, el análisis de contenido de ciertas normativas, manuales de puesto y documentación afín, en intersección con el acercamiento al grupo profesional que se encuentra laborando en orientación.

A partir de este posicionamiento, cada participante se selecciona a conveniencia, tomando como criterios básicos, entre ellos, que sean 
profesionales con nombramiento formal dentro del Ministerio de Educación Pública como orientadores u orientadoras en cualquiera de sus modalidades y que, al menos, posean 5 años de experiencia.

El estudio permite involucrar diferentes fases de exploración realizadas en este estudio: la fase de indagación referencial, la cual incluye la compilación de perfiles de contratación y documentos afines, como la jurisprudencia específica, la recopilación de los datos a través de entrevistas semiestructuradas (fase empírica).

Las personas investigadoras tuvieron acceso al campo, gracias al apoyo de las máximas autoridades del Ministerio de Educación Pública en materia de orientación, esto permite la apertura a la información, el encuentro constante que refuerza los resultados y el entendimiento de la dinámica especial que configura la disciplina de la orientación en este contexto, también se logra la sistematización y el análisis de resultados, mediante el CAQDAS (Computer Assisted Qualitative Data Analysis Sofware) denominado Atlas.Ti, para la codificación de datos en concordancia con las categorías e intersecciones planteadas (funciones, jurisprudencia y desempeño de la labor orientadora).

\section{Resultados, análisis y discusión}

El modelo institucional es particular, de acuerdo con las especificidades del centro educativo. La amplia oferta educativa determina el tipo de centro en el cual se encuentra el servicio de orientación; sin embargo, existe una homogenización de funciones que se van diversificando a través de lineamientos generales emanados del DOEV. En palabras de una de las personas participantes de la investigación,

Entonces habrá cosas que no se ajustan tanto a los equipos, como por ejemplo uno de los lineamientos que pide el DOEV es el expediente acumulativo, que realmente no se ajusta al equipo interdisciplinario, porque somos tres a veces viendo un caso, no es un expediente solo de orientación, entonces en ese sentido yo personalmente hago mi expediente y creo que lo hago muy bien e incluso me gusta hacerlo muy bien, pero no con todo los formatos que piden en el MEP, precisamente para que se ajusten más a la labor de nosotros, pero bueno tal vez en ese sentido se dan algunos ajustes, pero si se hacen. (Participante 3 ) 
A nivel disciplinar, existe claridad de las acciones del subsistema de centro educativo en primaria o secundaria en la población entrevistada; por ejemplo, la atención colectiva que se da en secundaria se entiende obligatoria y necesaria, mientras que la individualizada, por lo general, se da producto de la anterior y es generalizada en los datos obtenidos en el estudio; asimismo, es menos visible, aunque no ausente, la necesidad de programar atención grupal en aquellos casos donde estudiantes tengan situaciones parecidas y que de forma grupal se pueden ahondar a nivel de la orientación. Una de las participantes acota:

Cuando nosotros hablamos de atención individual es un tema interesantísimo en el MEP usted tiene que hacer magia, o sea no sabemos en qué momento un estudiante va a caer en crisis sea existencial, vocacional, o por terminar con la novia necesito contención y otras situaciones donde también como lo menciono el compañero el cyberbullying porque todos los centros educativos estamos bombardeados en este momento fuertemente de esta situación. (Participante 1)

El trabajo interdisciplinar es más visible en escuelas que cuentan con equipos interdisciplinarios, mientras que en otras escuelas y en los colegios que no los tienen, la interdisciplinariedad es vista de forma natural como aquella que se coordina entre docentes y la dirección del centro educativo; a nivel externo, también se dan vinculaciones tanto con instituciones que colaboran o con las que obligatoriamente hay que remitir situaciones relevantes del estudiantado, ya sea el PANI, los juzgados especializados en violencia, entre otros. Al respecto una de las participantes indica:

Atendemos los protocolos de suicidio, por ejemplo, o de "cutting", estos protocolos implican que se tiene que hacer una referencia al EBAIS y la referencia a los encargados de la persona menor de edad para que pida cita con el médico general e inicie un proceso hasta que llegué a psicología, a veces cuando llega a psicología resulta que la persona no quiere ir y los protocolos dicen que nosotros tenemos que darle seguimiento al caso, de saber que realmente fueron al hospital, de que tuvieron la cita, de que 
llegaron, que no lo dejaron botado, entonces tanto del hospital como de la escuela estamos en ese va y viene. (Participante 3 )

En el análisis de la categoría de la jurisprudencia, se generaliza el cumplimiento que estipula el título II del régimen de Carrera Docente que indica la clase de orientación obligatoria como parte de su función técnica-docente. Según los sujetos participantes de este estudio, queda claro dicho cumplimiento al indicar "lo primero que sale a relucir son la ejecución de los programas de estudio de orientación" (Participante 1). Sin embargo, en ocasiones se combina dicha función con las demandas que requiere el puesto en casos emergentes y urgentes:

Eso no se planea, muchas veces planeamos para mañana, esto y aquello, y si volvemos a ver la agenda para la tarde, no pudimos hacer lo que estaba planeado, por que surgieron cosas muy importantes, atención estudiantes, inclusive padres y madres de familia, que llegan en ese momento con una situación urgente que tenemos que atender. (Participante 2)

En concordancia con dicha categoría, en la escuela primaria, otra participante incorpora dentro de sus funciones la implementación de los protocolos para el suicidio, indica que el seguimiento a estos casos satura el tiempo laboral, y lleva a descuidar otras áreas, empero, para cumplir con los lineamientos utiliza como estrategia vincular las situaciones emergentes con los lineamientos, plantea que "del montón de emergentes, se hacen calzar con algún lineamiento" (Participante 3).

El trabajo cotidiano de orientación escolar está impregnado de situaciones emergentes, propio del trabajo con personas, es decir, seres volubles y cambiantes; tal y como lo señala la participante 3 a continuación:

Como se dan cuenta hay que tener buena memoria, ser muy ordenado y agendar las cosas, porque uno trae una planificación, o al menos eso es lo que hago yo todos los días, traigo mi plan y después de ahí viene todo lo demás producto de la sobrepoblación que atendemos, en mi caso ahorita estamos atendiendo alrededor de cuatrocientos cincuenta estudiantes cada orientador, entonces es bastante pesado. 
Es decir, además de la atención al estudiantado, a las madres y padres de familia, el trabajo interdisciplinario y las coordinaciones propias del trabajo; el reto mayor es el ratio de atención por cada profesional y, aún más grave en las escuelas, donde no existe ratio, sino la atención total del centro educativo.

\section{Conclusiones}

El modelo institucional de la orientación en el sistema educativo público costarricense se considera como un todo y sus subsistemas se catalogan en diferentes niveles para interactuar con el estudiantado y la atención a sus necesidades durante su desarrollo integral, este es el principal y se ubica en los centros educativos. El segundo subsistema se ubica en las Asesorías Regionales de Orientación (ARO), encargadas de promover y dar seguimiento de la implementación en relación con los lineamientos dictados desde las asesorías nacionales, así como de brindar acompañamiento técnico a su población meta. El último nivel piramidal del modelo se refleja en la asesoría nacional, que tiene entre sus tareas planificar, organizar, ejecutar, coordinar, asesorar, investigar, y evaluar las acciones estratégicas de la orientación educativa y vocacional en el Ministerio de Educación Pública.

El modelo institucional del servicio de orientación en el sistema educativo del Ministerio de Educación Pública es inconcebible sin el andamiaje legal que conforma el accionar de las modalidades, niveles y el contexto específico de cada centro educativo. Las demandas del ejercicio profesional, tanto por el cumplimiento de los lineamientos técnicos emanados del DOEV en el MEP, como por aquellos que surgen producto de las situaciones emergentes disciplinares de la cotidianidad laboral, hacen que el trabajo profesional tenga un alto volumen de labores que se ve permeado de situaciones emergentes propias del ámbito laboral con personas -mayoritariamente- en las etapas de la niñez y la adolescencia. Esto se explica, dado que se trabaja con el ser humano cuyas subjetividades lo hacen dinámico y complejo; no obstante, la labor en el MEP está cargada de proyectos, programas y algunas duplicidades en las funciones, que en ocasiones se vuelve una acción política de rendición de cuentas de itinerarios políticos.

Este escenario laboral involucra una serie de desafíos que van desde la atención individualizada estudiantil en sus diversas etapas de su desarrollo vital, hasta las propias de las coordinaciones entre las 
familias, las instancias que tienen en algunos casos relación con el estudiantado; asimismo, el trabajo interdisciplinario, las acciones propias de proyectos institucionales, comunales, regionales y el cumplimiento de las políticas nacionales del MEP. No siempre es posible contar con la sincronicidad en las intervenciones entre los subsistemas, y de cierta manera el modelo es un ideal, dado que la complementariedad entre demandas y las necesidades cotidianas de la labor representan el más grande reto profesional; por lo general, se priorizan las acciones en los procesos MEP y vinculantes de otras instituciones.

El modelo institucional cobra su particularidad en cada centro educativo, según las funciones técnico-docentes (secundaria) o técnico-administrativas (primaria); también la acción orientadora posee un trabajo interdisciplinar distinto, según la variedad de disciplinas profesionales con quienes comparte responsabilidades; ocurre esto de forma particular por la iniciativa histórica que se dio en Costa Rica, cuando el MEP promovió la contratación de profesionales en sicología, sociología o trabajo social, quienes en conjunto con el colectivo de profesionales de la orientación hicieran el trabajo interdisciplinario planteado para zonas que presentaban desventajas en sus índices sociales.

Se ha dejado en evidencia que existe carencia en la contratación de profesionales de orientación en el sistema educativo y que el ratio de atención a estudiantes es muy amplio en aquellos centros donde existen plazas asignadas a esta profesión, en comparación con las funciones que se desempeñan con otros parámetros sugeridos internacionalmente para la calidad de estos procesos. Es claro que, a mayor cantidad de profesionales que se nombran, mayor cobertura y, por ende, se pueden suplir mayores necesidades de atención a la población estudiantil.

El Servicio de Orientación en el MEP se ha implementado por años en el subsistema de centro educativo, como una estrategia que permite favorecer algunas acciones de prevención y de atención por parte del profesorado, por medio del Programa Guía y los Comités de Orientación, ante la ausencia de la persona profesional en orientación.

Es importante considerar que las demandas cotidianas en los subsistemas planteados superan el volumen de trabajo, por lo cual derivan en un desgaste profesional producto de esta condición. Al respecto, la coordinación y apoyo entre subsistemas son vitales para el desarrollo de la orientación en el sistema educativo costarricense. 


\section{Recomendaciones}

Se hace necesario el fortalecimiento de las vinculaciones entre subsistemas, para crear las alianzas y el trabajo estratégico entre los sistemas. Se presentan disposiciones ministeriales que impiden estas coordinaciones entre los niveles central, regional y de centro educativo que desfavorecen o impiden esta coordinación; ha quedado en evidencia que el volumen de trabajo producto de la cantidad de estudiantes y sus diversas necesidades hacen que el cumplimiento de las funciones de orientación, así como la calidad de logro de su objeto de estudio se vean limitadas, por ello este factor debe ser considerado por las autoridades del MEP, asimismo, la necesidad de considerar más códigos de plazas en orientación en aquellos centros educativos especialmente de educación primaria, que aún no cuentan con este tipo de profesionales.

A partir del modelo institucional del sistema educativo, es necesario fortalecer las construcciones teóricas que están permeando la labor orientadora en la cotidianidad profesional; los fundamentos epistemológicos de la orientación educativa deben enfatizar en las teorías educativas y de desarrollo humano, y no solo enfatizar en los enfoques psicologistas, que también son de gran valor; por ello, la educación superior puede establecer esta revisión constante dentro de su quehacer, con el fin de coadyuvar a la orientación como disciplina y profesión. Además, se deben incorporar elementos, herramientas y recursos que le permitan a este grupo profesional conocer y conocerse en un campo laboral tan cambiante y demandante.

Existen diferencias entre la concepción de modelo institucional de la categoría modelo organizacional; el primero corresponde a instituciones de orden público y estatal y el segundo al orden privado. Por ello, pese a que existe libertad en las visiones y misiones de las instituciones gubernamentales y en las no gubernamentales, es importante que, desde el Colegio de Profesionales de Orientación, se siga discutiendo el objeto de estudio de la orientación y las implicaciones epistemológicas, axiológicas, ontológicas y metodológicas que delimitan su accionar de otras disciplinas y consolidar su identidad profesional. 


\section{Referencias}

Arencibia, J. S. (2012). Modelos de orientación e intervención psicopedagógica. Servicio de Publicaciones y Difusión Científica de la ULPGC.

Asamblea Legislativa. (1966). Convenio Centroamericano sobre Unificación Básica de la Educación. Recuperado de https://bit. ly/30eahiK

Carvajal, J. A., Estrada, M. A., Mora, G. M. y Vargas, S. (2014). Modelo de intervención de orientación en el sistema educativo costarricense. San José: Ministerio de Educación Pública. Dirección de Vida Estudiantil. Departamento de Orientación Educativa y Vocacional.

Dirección General de Servicio Civil, Costa Rica. (2020). Manual de clases actualizado al 27-05-2020. Recuperado de https://bit. ly/30eZBR2

Fung-Lung, M. (2017). Las funciones que desempeñan el personal de orientación y sus implicaciones jurídicas en la prestación de los servicios educativos. Revista Gestión de la Educación, 7(1), 3772. Recuperado de https://bit.ly/2YyaTis

Gamboa, A., Álvarez, N. M., Flores, P. E., Miranda, K. F. y Vindas, K. A. (marzo, 2018). El síndrome de burnout en profesionales de la orientación en secundaria. Trabajo presentado en el II Congreso Profesionales en Orientación. San José, Costa Rica. Resumen recuperado de https://bit.ly/2YKTzqI

Gamboa, A. y Fallas, M.A. (2019). Informe final. Proyecto modelos de orientación: Una mirada desde el desempeño profesional. Heredia: Sistema de Información Académica, División de Educación para el Trabajo, Centro de Investigación y Docencia en Educación. Universidad Nacional.

Grañeras, M. y Porras, A. (2008). Orientación educativa: Fundamentos teóricos, modelos institucionales y nuevas perspectivas. Ministerio de Educación, política social y Deporte. España: Omagraf.

Ministerio de Educación Pública. (2019). Organización técnico-administrativa para operacionalizar el Servicio de Orientación en el ámbito regional y de centro educativo, 2019. Oficio DVEDOEV-74-2019, San José, 28 de enero de 2019. 
Ministerio de Educación Pública. (2017a). Objeto de estudio. San José, Costa Rica: Ministerio de Educación Pública.

Ministerio de Educación Pública. (2017b). Programas de Estudio de Orientación primero, segundo y tercer ciclos de la Educación General Básica y Educación Diversificada. San José, Costa Rica: Ministerio de Educación Pública.

Ministerio de Educación Pública. (2017c). Compendio de las ofertas y servicios del Sistema Educativo Costarricense 2016. San José, Costa Rica: Dirección de Planificación Institucional, Ministerio de Educación Pública.

Ministerio de Educación Pública. (2011). Tabla de recursos de los orientadores y orientadores asistentes de los centros educativos. Oficio DPI-DDSE-0296-11, San José, 03 de agosto de 2011.

Monescillo, M, Méndez, J y Bisquerra, R. (2008). Orígenes y desarrollo de la orientación. modelos de orientación e intervención psico-pedagógica. Barcelona: Praxis.

Monescillo, M, Méndez, J y Bisquerra, R. (2011). Orígenes y desarrollo de la orientación. En R. Bisquerra (Coord.), Modelos de orientación e intervención psicopedagógico (pp. 23-38). Madrid: Wolters Kluwer España, S.A.

Müller, M. (1998). Descubrir el camino, nuevos aportes educacionales y clínicos de orientación vocacional. Buenos Aires: Bonum.

Müller, M. (2004). Subjetividad y orientación vocacional profesional. Orientación y Sociedad, 4, 5-44. Recuperado de https://bit. ly/2yhyx 81

Naranjo, M. L. (2004a). Enfoques humanistico-existenciales y un modelo ecléctico. San José, Costa Rica: Editorial de la Universidad de Costa Rica.

Naranjo, M. L. (2004b). Enfoques conductistas, cognitivos y racional emotivos. San José, Costa Rica: Editorial de la Universidad de Costa Rica.

Asamblea Legislativa. (1966). Convenio Centroamericano sobre Unificación Básica de la Educación. Recuperado de https://bit. ly/3fkM1Rd

Asamblea Legislativa. (1957). Ley Fundamental de Educación, 25 de setiembre de 1957. Recuperado de https://bit.ly/3aZhpRP

Poder Ejecutivo. (1996). Funciones encomendadas a los profesionales del Estrato Técnico Docente. La Gaceta N. ${ }^{\circ} 220$ del 15 de noviembre de 1996. 